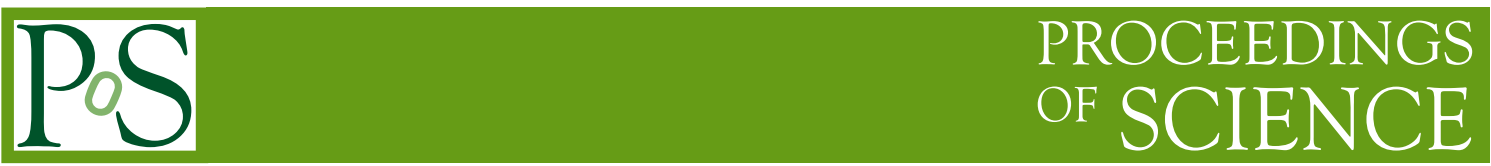

\title{
Evolution of Planetary Nebulae and PAH features
}

\author{
Ryou Ohsawa ${ }^{* a}$, Takashi Onaka ${ }^{a}$, Itsuki Sakon ${ }^{a}$, Tamami I. Mori ${ }^{a}$, Issei Yamamura ${ }^{b}$, \\ Mikako Matsuura $^{c}$, Hidehiro Kaneda $^{d}$, Jeronimo Bernard-Salas $^{e}$, Olivier Berné ${ }^{f}$, \\ and Christine Joblin $f$ \\ Graduate School of Science, University of Tokyo, 113-0033 Tokyo, Japan ${ }^{a}$ \\ ISAS/JAXA, 252-5210 Kanagawa, Japan ${ }^{b}$ \\ University College London, Gower St, London WC1E 6BT, United Kingdom ${ }^{c}$ \\ Nagoya University, 466-8550 Aichi, Japan ${ }^{d}$ \\ Open University, Walton Hall, Milton Keynes, Buckinghamshire MK7 6AA, United Kingdome \\ IRAP Université de Toulouse \& CNRS, 9, Av. du Colonel Roche BP 44346 F-31028 Toulouse \\ Cedex 4, France ${ }^{f}$ \\ E-mail: ohsawaeastron.s.u-tokvo.ac.jp
}

\begin{abstract}
Near- to mid-infrared spectra of 17 Galactic Planetary Nebulae (PNe), which show prominent C-rich dust features, were obtained with the AKARI/IRC and Spitzer/IRS. Relative intensities of Polycyclic Aromatic Hydrocarbon (PAH) features were measured. The evolutionary stage of PNe is estimated by the effective temperature of the central star. The evolution of the PAH emission is investigated in terms of the evolution of the central stars. As PNe evolve, the ionization fraction of PAHs decreases, the 3.4-3.5 $\mu \mathrm{m}$ aliphatic features become stronger and the peak position of the $6.2 \mu \mathrm{m}$ feature moves toward shorter wavelengths. Possible mechanisms for the evolution of the PAH emission are discussed.
\end{abstract}

The Life Cycle of Dust in the Universe: Observations, Theory, and Laboratory Experiments - LCDU 2013, 18-22 November 2013

Taipei, Taiwan

\footnotetext{
* Speaker.
} 


\section{Background}

Infrared broad emission bands are widely observed in a variety of objects in the Universe. They are usually referred to as the unidentified infrared (UIR) bands. Several candidates have been proposed as the carriers of the UIR bands [ [6, 目, 四]. The most famous candidate is polycyclic aromatic hydrocarbons (PAHs), which are made of fused aromatic rings with hydrogen atoms. Due to their small size, PAHs are stochastically excited by a single UV-photon, pushing them into a vibrational mode that corresponds to a specific infrared emission band.

As with other dust grains, it is widely believed that PAHs are formed in the ejecta of evolved stars and eventually injected into interstellar space. The properties of PAHs may change if the PAHs are processed. Whether PAHs are processed around evolved stars should be examined to understand what kinds of dust grains are sent into interstellar space.

Several studies have investigated the evolution of the PAH emission during the post-asymptotic giant branch (AGB) phase. Systematic variations in the peak positions of the 11.3 and $7.7 \mu \mathrm{m} \mathrm{PAH}$ features are reported along with the effective temperature of the central star [ [ $8, \mathbb{1}]$ ]. The results suggest that PAHs may be processed during the post-AGB phase. However, there are few studies on the evolution of the PAH emission during the planetary nebula (PN) phase, which follows the post-AGB phase. We investigate the evolution of the $\mathrm{PAH}$ emission during this later stage.

The major PAH features are located around 3-14 $\mu \mathrm{m}$. In this work, the near- to mid-infrared continuous spectra of Galactic PNe are collected. The near-infrared part $(2-5 \mu \mathrm{m})$ is obtained with AKARI/IRC, while the mid-infrared part $(5-14 \mu \mathrm{m})$ is obtained with Spitzer/IRS. Since the IRS slit is narrower than that of IRC, the IRS spectra are scaled to match up with the IRC counterparts. Since the effective temperature of PNe monotonically increases with evolution [व], we use it as an indicator of the PN's evolutionary phase. These effective temperatures have been collected for a total of 17 objects from the literature.

The intensities of the PAH features at 3.3, 3.4-3.5, 7.7, and $11.3 \mu \mathrm{m}$ are measured by spectral fitting with Lorentzian profiles $\left(I_{3.3}, I_{3.4}, I_{7.7}\right.$, and $I_{11.3}$ respectively). The peak wavelength of the $6.2 \mu \mathrm{m}$ PAH feature $\left(\lambda_{6.2}\right)$ is also measured by fitting.

\section{Results \& Discussion}

Figure $\square$ shows the variations in the PAH emission along with the effective temperature: panel $A$ shows the evolution of the $I_{7.7} / I_{11.3}$ ratio, indicating the PAH ionization fraction [⿴囗十); panel $B$ shows the $I_{3.3} / I_{11.3}$ ratio, indicating the relative amount of small-sized PAHs [ए2] ; the $I_{3.4} / I_{3.3}$ ratio is shown in panel $C$, indicating the relative amount of aliphatic components in PAHs [[]]; panel $D$ shows the peak wavelength $\lambda_{6.2}$, indicating the PAH emission class [Q]. These show systematic changes with the effective temperature at confidence levels higher than $90 \%$. The present results indicate that the PAH ionization fraction increases, the amount of small-sized PAHs decreases, the amount of aliphatic component increases, and the $6.2 \mu \mathrm{m}$ peak position moves toward shorter wavelengths as the PN evolves.

Are these variations caused by processing of PAHs? The PAH ionization fraction, which is controlled by $G_{0} T^{\frac{1}{2}} n_{\mathrm{e}}^{-1}$, where $G_{0}$ is the radiation field intensity, $T$ is the gas temperature, and $n_{\mathrm{e}}$ is the electron density [B]], is not directly related to processing of PAHs. Small-sized PAHs are broken 


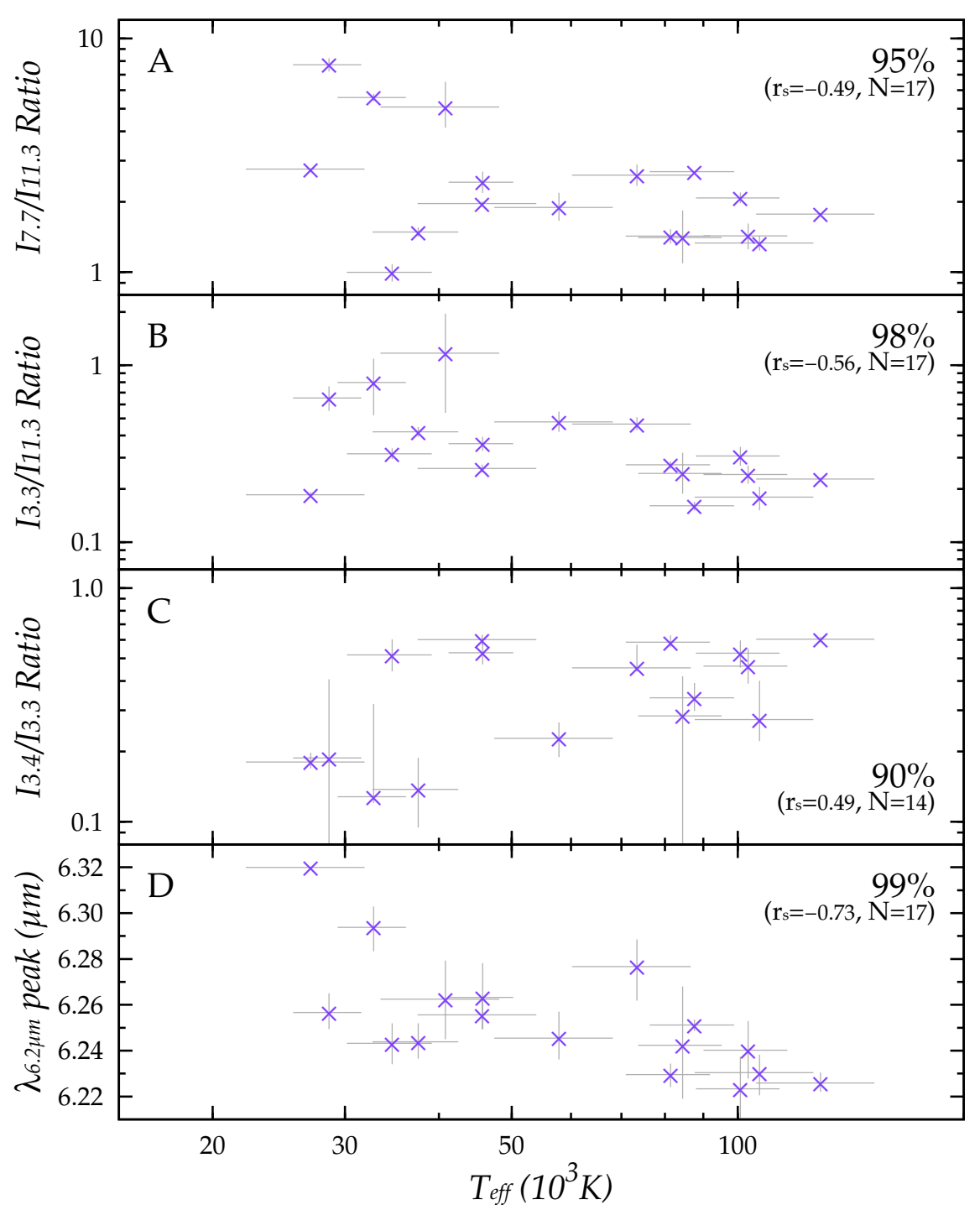

Figure 1: Evolution of the PAH emission with the effective temperature. $A$ : the $I_{7.7} / I_{11.3}$ ratio, indicating the PAH ionization fraction; $B$ : the $I_{3.3} / I_{11.3}$ ratio, indicating the fractional amount of small PAHs; $C$ : the $I_{3.4} / I_{3.3}$ ratio, indicating the amount of aliphatic components in PAHs; $D$ : the peak wavelength of the $6.2 \mu \mathrm{m}$ feature, indicating the PAH emission class $[Q]$. The numbers in each panel refer to the confidence level, with which the systematic variation with the effective temperature is significant. $r_{\mathrm{s}}$ is the Spearman's correlation coefficient and $N$ is the number of objects with a sufficient signal-to-noise ratio.

easily [四, while aliphatic components are more fragile than aromatic components. The amount of small-sized PAHs and aliphatic components can thus be used as sensitive indicators of PAH processing. Photo-dissociation of PAHs may be the most effective process in a PN environment. When photo-dissociation of PAHs is promoted, both the amount of small-sized PAHs and aliphatic components likely decrease. However, processing of PAHs does not explain the decrease in the $I_{3.3} / I_{11.3}$ ratio and the increase in the $I_{3.4} / I_{3.3}$ ratio at the same time. We therefore conclude that the observed variations in the PAH emission during the PN phase is not attributed to processing of PAHs. 
Instead of PAH processing, we propose that the evolution of the PAH emission is brought about by the expansion of the photo-dissociation region (PDR). The observed PAH emission comes only from PAHs excited in the PDR. Assuming that the properties of PAHs, such as the size distribution and the amount of aliphatic components, vary with distance from the central star, the averaged properties of PAHs in the PDR may change with its expansion. The evolution of the PAH emission may be attributed to the variation in the PAH properties along the radial direction. This hypothesis will be examined in future investigation.

This research is in part based on observations with AKARI, a JAXA project with the participation of ESA and on archival data obtained with the Spitzer Space Telescope, which is operated by the Jet Propulsion Laboratory, California Institute of Technology under a contract with NASA. This is supported in part by a Grant-in-Aid for Scientific Research (25-8492) by the Japan Society of Promotion of Science.

\section{References}

[1] Allain, T., Leach, S., and Sedlmayr, E., Photodestruction of PAHs in the interstellar medium. I. Photodissociation rates for the loss of an acetylenic group, A\&A, 305, 602 (1996)

[2] Blöcker, T., Stellar evolution of low- and intermediate-mass stars. II. Post-AGB evolution, A\&A, 299, $755,(1995)$

[3] Bregman, J. and Temi, P., Variations of the Mid-Infrared Emission Spectrum in Reflection Nebulae, ApJ, 621, 831, (2005)

[4] DeFrees, D. J., Miller, M. D., Talbi, D., Pauzat, F., et al., Theoretical infrared spectra of some model polycyclic aromatic hydrocarbons - Effect of ionization, ApJ, 408, 530, (1993)

[5] Joblin, C., Tielens, A. G. G. M., Allamandola, L. J., Geballe, T. R., Spatial Variation of the 3.29 and 3.40 Micron Emission Bands within Reflection Nebulae and the Photochemical Evolution of Methylated Polycyclic Aromatic Hydrocarbons, ApJ, 458, 610, (1996)

[6] Jones, A. P., Variations on a theme - the evolution of hydrocarbon solids. II. Optical property modelling - the optEC $C_{(s)}$ model, $A \& A, \mathbf{5 4 0}, \mathrm{A} 2,(2012)$

[7] Kwok, S. and Zhang, Y., Mixed aromatic-aliphatic organic nanoparticles as carriers of unidentified infrared emission features, Nature, 479, 80, (2011)

[8] Matsuura, M., Bernard-Salas, J., Lloyd Evans, T., and Volk, K. M., et!al., Spitzer Space Telescope spectra of post-AGB stars in the Large Magellanic Cloud - polycyclic aromatic hydrocarbons at low metallicities, MNRAS, in press.

[9] Peeters E., Martín-Hernández, N. L., Damour, F., Cox, P., et al., and et. al., The rich 6 to $9 \mu \mathrm{m}$ spectrum of interstellar PAHs, A\&A, 390, 1089, (2002)

[10] Sakata, A., Wada, S., Tanabé, T., and Onaka, T., Infrared spectrum of the laboratory-synthesized quenched carbonaceous composite (QCC) - Comparison with the infrared unidentified emission bands, ApJ, 287, L51, (1984)

[11] Sloan, G. C., Jura, M., Duley, W. W., Kraemer, K. E., et al., The Unusual Hydrocarbon Emission from the Early Carbon Star HD 100764: The Connection between Aromatics and Aliphatics, ApJ, 664, $1144,(2007)$

[12] Schutte, W. A., Tielens, A. G. G. M., and Allamandola, L. J., Theoretical modeling of the infrared fluorescence from interstellar polycyclic aromatic hydrocarbons, ApJ, 415, 397, (1993) 